\title{
Author Correction: Portraits of physicists
}

Marco Bentivegna, Richard Brierley and Nina Meinzer

Correction to: Nature Physics https://doi.org/10.1038/nphys4324, published online 5 December 2017.

In this Books \& Arts, the surname of the first author was spelt incorrectly; it should have read 'Bentivegna'.

Published online: 20 February 2019

https://doi.org/10.1038/s41567-019-0456-1 